\title{
Dissecting the bulge in hair regeneration
}

Peggy Myung ${ }^{1}$ and Mayumi Ito²

\author{
1Department of Dermatology, University Hospitals Case Medical Center, Case Western Reserve University, Cleveland, Ohio, USA. \\ 2Departments of Dermatology and Cell Biology, New York University School of Medicine, New York, New York, USA.
}

\begin{abstract}
The adult hair follicle houses stem cells that govern the cyclical growth and differentiation of multiple cell types that collectively produce a pigmented hair. Recent studies have revealed that hair follicle stem cells are heterogeneous and dynamic throughout the hair cycle. Moreover, interactions between heterologous stem cells, including both epithelial and melanocyte stem cells, within the hair follicle are just now being explored. This review will describe how recent findings have expanded our understanding of the development, organization, and regeneration of hair follicle stem cells. At a basic level, this review is intended to help construct a reference point to integrate the surge of studies on the molecular mechanisms that regulate these cells.
\end{abstract}

\section{Introduction}

Throughout an individual's lifetime, epidermal cells of the skin terminally differentiate and migrate to the superficial layer of the epidermis, where they are shed, and are continuously replaced by proliferating germinative cells of the basal layer $(1,2)$. By contrast, the hair follicle regenerates through programmed phases of organized growth (anagen), regression (catagen), and rest (telogen). This cyclical growth and differentiation of multiple cell types that collectively produce a pigmented hair shaft is governed by resident stem cells, which reside in an area of the hair follicle called the bulge $(3,4)$.

The presence and function of hair follicle stem cells (HFSCs) are directly related to hair growth, and their absence results in hair loss. The ability to consistently regenerate this complex organ has made HFSCs the consummate model to study the mechanisms regulating adult stem cell maintenance, growth, and differentiation. Similar to many other somatic stem cells, HFSCs are selfrenewing and multipotent, possessing the ability to regenerate all epithelial layers of the hair follicle throughout life (5-7). Additionally, they hold plasticity and can differentiate into interfollicular epidermal cells during wound reepithelialization (8-11).

Stem cells are essential for maintaining the skin's integrity during homeostasis and in response to injury (8). Thus, characterization of these cells and the signals that regulate their quiescence and activation has become vital to translational studies and their clinical applications. The recent acceleration of progress in HFSC biology has been largely driven by pioneering studies that characterized the location and expression of molecular markers of this population (12-14). Since then, the growing number of identified stem cell markers has contributed to a more detailed recognition of the heterogeneity of the bulge population (15-20), and it is now evident that the HFSC niche is heterogeneous and dynamic throughout the hair cycle. Furthermore, the function and behavior of each subpopulation are differentially regulated during homeostasis and in response to injury $(16,21-24)$. We are now beginning to develop the genetic tools to examine these specific hair follicle populations in an effort to characterize their regulation and how they contribute to the pleiomorphic functions of HFSCs in hair regeneration, wound repair, and tumorigenesis. This review will provide con-

Conflict of interest: Mayumi Ito is listed as an inventor on a patent application related to wounding-induced hair follicle neogenesis owned by the University of Pennsylvania and has equity in Follica, a start-up company that has licensed the patent. Citation for this article: J Clin Invest. 2012;122(2):448-454. doi:10.1172/JCI57414. text for understanding some of the key concepts in HFSC biology and describe how recent findings have expanded our knowledge of how the HFSC niche is organized.

\section{The morphologic and kinetic definition of the bulge}

The cyclical growth of the hair follicle is maintained by multipotent stem cells that lie within a region called the bulge, generally located at the base of the permanent portion of the follicular outer root sheath (ORS) $(3,12)$. The origin of the term "bulge" arguably dates back to observations made in 1876 by Paul Gerson Unna, who described an epithelial swelling ("wulst") in the ORS of developing human hair follicles that is apparent in the embryo but inconspicuous in human adult hair follicles $(3,25$, 26). Others observed that the postnatal anagen hair follicle is derived from the epithelial (germ) sac, which consists of epithelial cells that surround the telogen club hair (25-28). Unlike adult human hair follicles, adult mouse telogen club hair follicles are retained and rest juxtaposed to the next growing anagen follicle. On histological sections, these cells can also create a bulge-like protrusion in the ORS of anagen hair follicles. This outward protrusion of the epithelial sac in mouse follicles is also now commonly referred to as the bulge (12).

The initial study that suggested the presence of epithelial stem cells in the bulge region of the hair follicle utilized kinetic studies to identify slow-cycling cells that can retain a nucleotide label $\left(\left[{ }^{3} \mathrm{H}\right]\right.$-thymidine or BrdU) following a long chase period (ref. 12 and Figure 1A). The ability to cycle slowly while maintaining high proliferative potential had been considered an essential characteristic of epithelial stem cells. This study revealed that slowly cycling label-retaining cells (LRCs) are located in the bulge region (12). It also served as the basis for the "bulge-activation hypothesis," which states that LRCs reside in the bulge and are activated through interactions with the adjacent dermal papilla (DP) (12). These cells give rise to rapidly proliferating transit-amplifying (TA) cells that migrate down to regenerate the lower cyclical portion of the follicle. According to the bulge-activation hypothesis, cells below this label-retaining region degenerate during catagen phase.

This work served as a breakthrough in HFSC biology; not only was it the first to localize HFSCs to a region that had been unappreciated since the studies of Unna, but it also provided the first means to histochemically identify the bulge region by label retention. However, at the same time, this terminology has evolved circularly, and "bulge" now commonly refers to the area in which HFSCs reside, regardless of the presence or absence of a morphological bulge. Unlike the telogen bulge, which can be clearly 
A

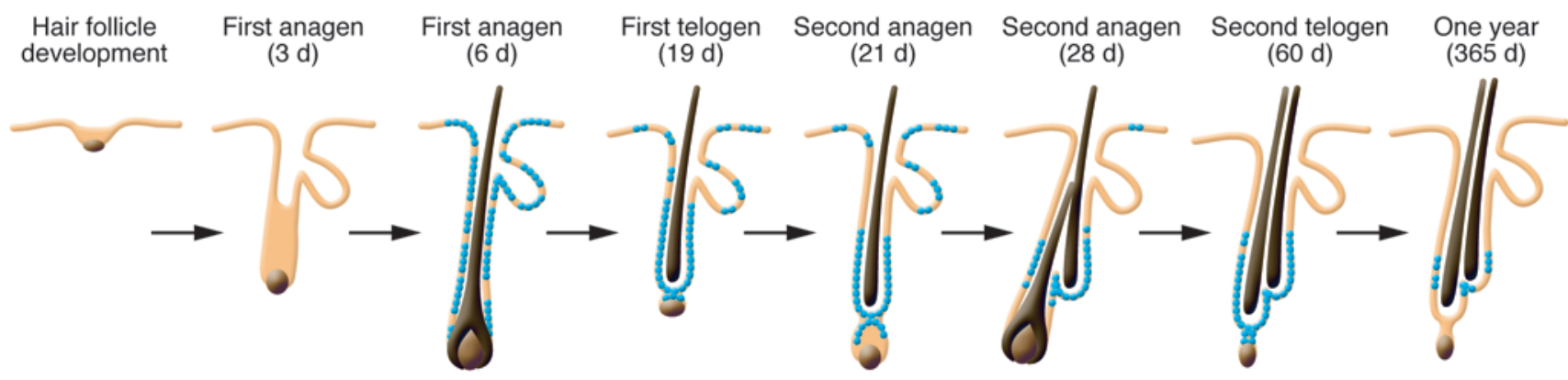

BrdU injection

Label chase

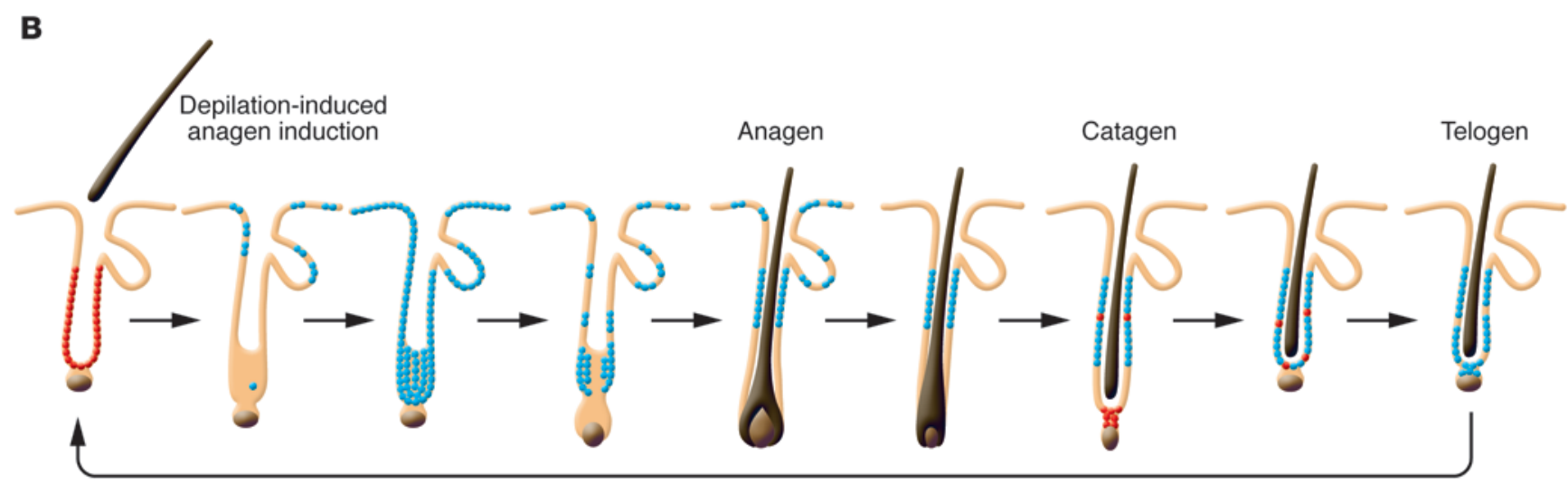

BrdU injection

Label chase

Cells with BrdU nucleotide

Apoptotic cells

DP

Hair shaft

\section{Figure 1}

The location of LRCs during the hair cycle. (A and B) During spontaneous anagen phase (A) or depilation-induced anagen phase (B), actively dividing sHG and bulge cells incorporate a pulse of BrdU nucleotide label. During anagen phase, cells within the sHG/bulge cycle infrequently and give rise to rapidly proliferating TA cells that migrate down to the bulb, which contains matrix cells. In contrast, slow-cycling cells from the $\mathrm{sHG} /$ bulge area retain the BrdU label and remain in the telogen sac and upper portion of the anagen ORS. During the chase period, rapidly dividing cells lose the BrdU label and, by the late anagen phase, can no longer be detected, while those slow-cycling cells in the upper hair follicle retain the BrdU label (LRCs). During the catagen phase, cells within the matrix and lower ORS undergo apoptosis, and the hair follicle retracts upward with the associated DP. LRCs remain as the permanent population of the ORS and can also be seen in the epithelial strand during the catagen phase. As the catagen phase proceeds, LRCs in the forming club hair follicle begin to upregulate bulge and sHG markers while entering the telogen phase. In the telogen phase, the sHG rests above the DP, while the bulge surrounds the club hair as an epithelial sac. (B) In depilation-induced anagen phase, nearly all bulge cells undergo apoptosis after the club hair is plucked and are quickly replenished by remaining proliferating sHG cells. As sHG cells repopulate the bulge, cells within the bulge region upregulate bulge-specific markers.

identified as the epithelial sac surrounding the club hair, the lowermost permanent portion of the adjacent anagen ORS does not show any protrusion histologically. Therefore, the precise border between anagen bulge cells and other ORS cells cannot be defined morphologically. Label-retention assays can be used to define the region of relatively slow-cycling cells, but the exact length of the label-retaining region in the ORS is a function of the time and duration of nucleotide pulse. Consequently, the border between slow-cycling stem cells and more proliferative ORS cells is not identical between studies.

\section{Resolution of the telogen bulge}

Following the adoption of the bulge-activation hypothesis, telogen bulge cells were found to express specific markers, including keratin-15 (K15) $(13,29)$ and CD34 (14). This early characterization paved the way toward isolating and investigating HFSCs in detail. Specifically, in vivo genetic lineage tracing experiments have shown that all layers of the lower portion of the anagen hair follicle are derived from $\mathrm{K}^{+} 5^{+} \mathrm{HFSCs}(6)$. Additionally, ex vivo transplant and grafting experiments using purified bulge cells demonstrated that these cells have the potential to differentiate into all skin epithelial lineages (5-7). Since then, the growth of data in this field continues to offer new insight into the regulation of HFSCs at the molecular level. However, it is still unclear whether every cell within the telogen sac holds the capacity to differentiate into all eight epithelial cell types that compose the anagen hair follicle in vivo, or whether specific subpopulations have restricted lineage commitment and/or self-renewing capacity (3). 


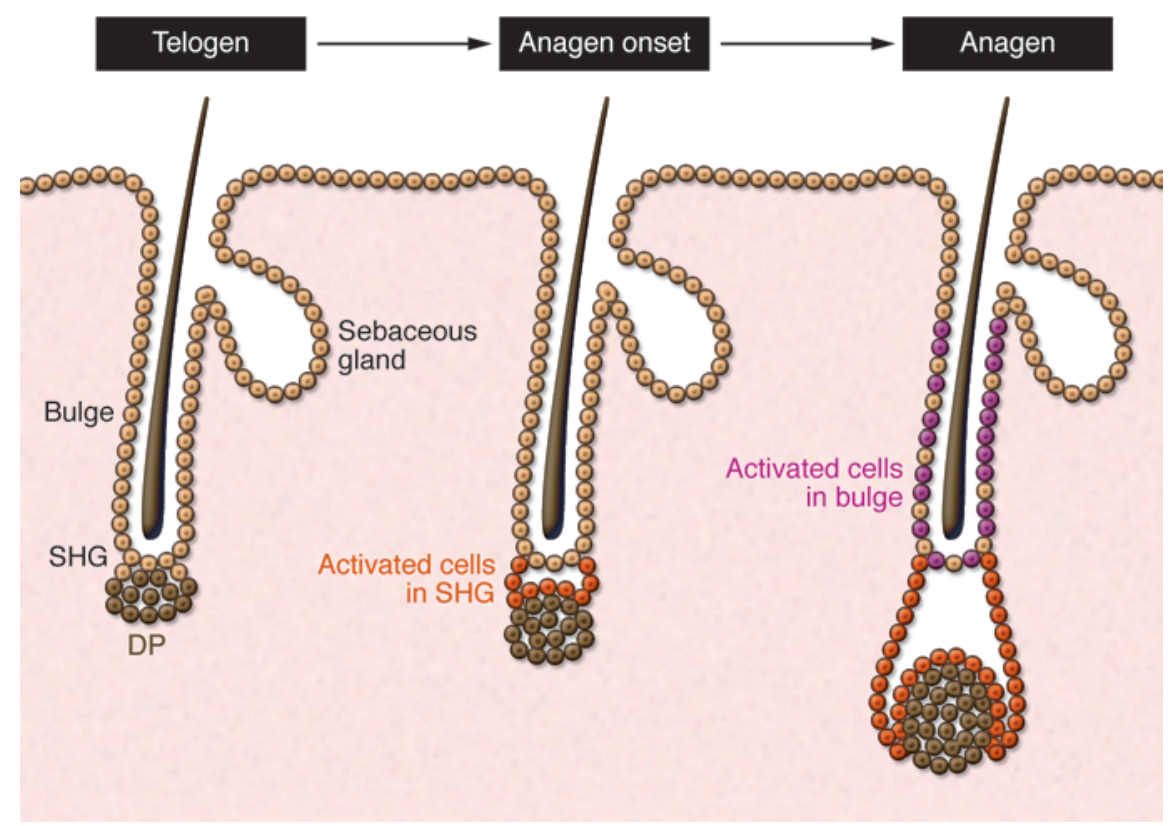

\section{Figure 2}

Two-step mechanism of activation. The sHG and bulge cells are biochemically and functionally distinct populations (35). During late telogen/anagen onset, sHG cells respond first (orange) to signals from the DP to undergo activation and proliferation, which initiates hair growth during the anagen phase. The bulge cells respond by undergoing activation and proliferation later, during the anagen phase (purple). sHG cells are proposed to jump start hair growth, while bulge cells continue to cycle slowly during the anagen phase to extend the ORS and maintain the matrix to support hair growth.
In the past, the term "bulge" often included an anatomically close but distinct cell population that sits directly above the DP in the telogen follicle and has been called the secondary hair germ (sHG), named for its morphological and functional resemblance to the embryonic hair germ (30-32). Immunohistochemical analysis using markers such as CD34 and the calcium-binding proteins S100A4 and S100A6 demonstrated that the sHG represents a population that is biochemically distinct from neighboring bulge cells $(14,22,33,34)$. During anagen, sHG and bulge cells proliferate, and the sHG becomes morphologically indistinct as the hair follicle grows downward with the DP.

Recently, Greco et al. showed that sHG cells are selectively activated before bulge cells to initiate hair growth (Figure 2 and ref. 35). In this study, sHG cells proliferate first, during late telogen, whereas bulge cells proliferate later, during anagen. In addition, late-telogen sHG cells show molecular evidence of activation and a gene expression profile similar to that seen in bulge cells in anagen. Collectively, these data suggest that sHG cells represent an activated stem cell population that is primed to initiate hair growth in response to anagen stimuli derived from the DP (Figure 2). Similar changes occur later in bulge cells following anagen onset, possibly to sustain hair follicle growth throughout anagen. Incidentally, although described as occurring in late telogen, it has become more difficult to precisely define the specific hair cycle stage during which the sHG becomes activated. According to some studies, "late telogen" may also be considered "early anagen," since anagen onset can be characterized as the time when cells proliferate to produce hair (30). Nevertheless, another group also proposed a model in which bulge cells migrate to the sHG to become committed TA cells prior to anagen, reinforcing the concept that telogen is not static and instead is characterized by several changes that occur throughout that phase (36).

Still, no study has determined whether the bulge and sHG populations hold unique lineage commitment potentials. In particular, the fate of sHG cells during anagen and their self-renewing capacity remain unclear $(34,35)$. Although cells of the bulge region have been shown to generate all cells of the hair follicle, sebaceous gland, and epidermis in a reconstitution model $(5,6)$, the lack of genetic studies that independently label and track sHG and bulge cell populations has precluded in vivo lineage analysis of their respective contributions to the hair follicle during anagen and the epidermis following wounding. Lineage-tracing experiments using K15 or Lgr5 promoters, which are active in both the bulge and sHG, have only demonstrated the fate of these populations collectively $(6,16)$. Importantly, sHG cells have the ability to regenerate functional bulge cells upon depilation-induced bulge cell death $(22,34)$. The signals that allow sHG cells to transform into bulge cells are unknown, but it may be inferred that sHG cells hold stem cell potential. In addition to HFSCs, melanocyte stem cells (McSCs) also reside in the sHG and bulge, where they are coordinately activated with follicular stem cells during the anagen phase to generate mature bulb melanocytes (37). Examining the differential regulation of sHG and bulge McSCs will lend unique insight into our understanding of how the niche influences stem cell behavior to produce pigmented hair.

\section{Regeneration of the bulge and SHG}

Early studies utilized classic BrdU pulse-chase experiments to examine the morphogenesis of the telogen bulge and sHG during the catagen transformation phase, following depilation-induced anagen phase (Figure 1B) (34). BrdU administration during early anagen phase resulted in label uptake by virtually all hair follicle epithelial cells. As the anagen phase progressed, the label was limited to upper-to-mid ORS cells, largely corresponding to the bulge region, but eventually became undetectable in rapidly proliferating cells of the lower hair follicle, including matrix cells. By the following telogen phase, the telogen bulge and sHG mostly consisted of LRCs. This study showed that SHG and bulge cells of the telogen follicle are regenerated by LRCs of the anagen follicle (34).

This finding was confirmed by a later independent study that carefully examined the spontaneous hair cycle (35). In a more recent paper by Hsu et al., "bulge" referred exclusively to cells at or above the level of the epithelial sac of the club hair follicle (38). ORS LRCs below this point in the adjoining anagen follicle, which 


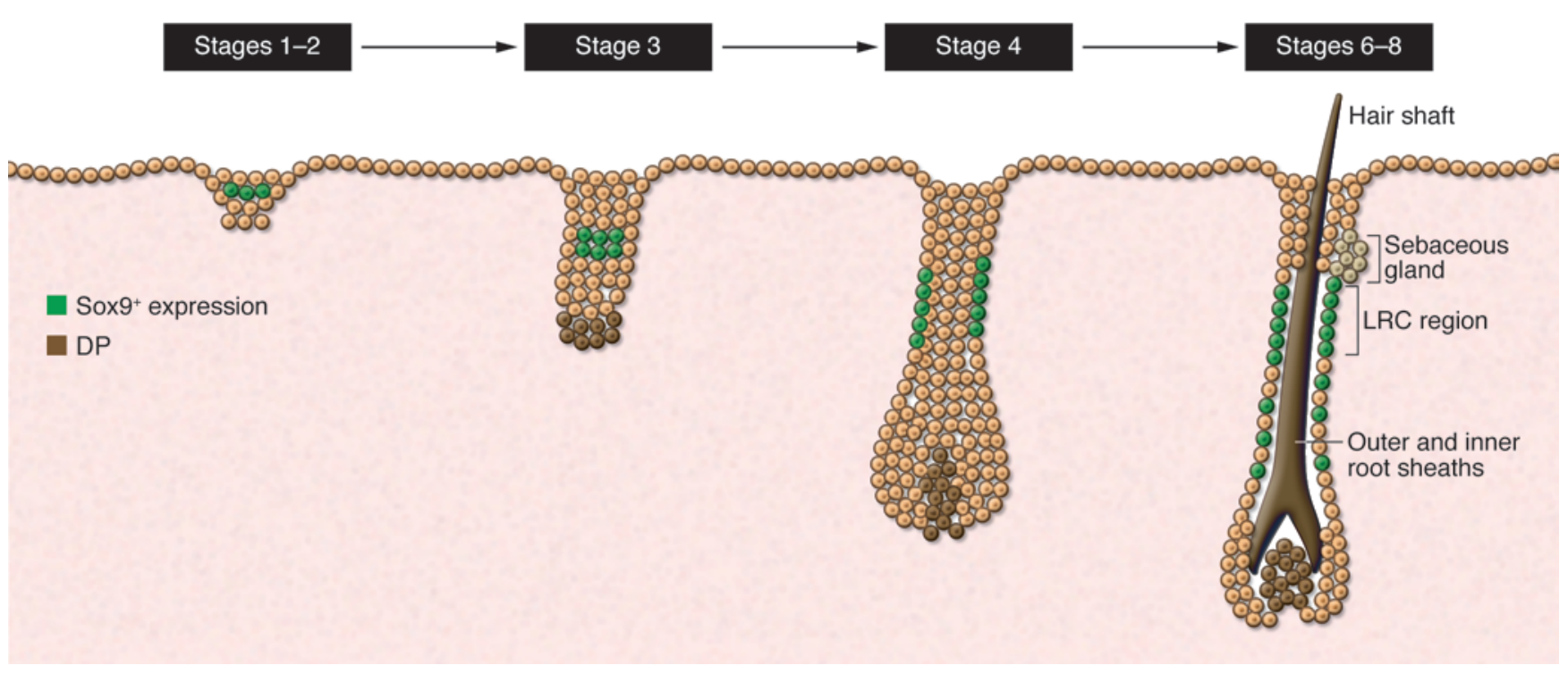

Figure 3

The genesis of a bulge stem cell population during embryonic hair development. At the placode/germ stage of hair development, cells within the suprabasal layer of the forming germ express some bulge-specific markers, including Sox9 (18). Slow-cycling LRCs, representing HFSCs in the adult hair follicle, can be identified in the upper ORS of developing hair follicles as they grow downward. These are Sox9+ and express other bulge markers (e.g., Tcf3, Lhx2, Nfatc1). They can be traced to the first postnatal telogen bulge/sHG and therefore form the first telogen bulge stem cell population. Sox9-derived cells can be found in the lower ORS and matrix during hair development. Similar to the function of bulge cells during postnatal anagen phases proposed by Greco et al. (35), embryonic Sox9+ putative stem cells are also thought to provide cells to support the matrix during hair growth.

had been included as part of the bulge in previous studies, were described by Hsu et al. as slow-cycling ORS cells that formed the bulge and most of the sHG of the new telogen follicle (38). Regardless of the terminology used, these studies showed that LRCs of the anagen hair follicle persist during catagen and form the subsequent telogen bulge and sHG, not only after depilation-induced anagen, but also during the spontaneous hair cycle $(34,35)$. Importantly, this study (38) also demonstrated that some proliferating cells of the lower anagen ORS can survive and return to the telogen follicle. These terminally differentiated cells lack stem cell potential and form a specialized postmitotic $\left(\mathrm{K}^{+}\right)$inner layer of cells that surround the dead telogen club hair and anchor it to the follicle. However, this $\mathrm{K}^{+}$layer also appears to provide paracrine signals that promote HFSC quiescence during telogen (38). Identification of promoters specific for this population will be valuable to further delineate how these cells function as extrinsic regulators of HFSCs.

\section{An embryonic wulst in mice}

As a morphological bulge is not evident in the mouse embryo, and hair follicle-restricted $\mathrm{K}^{+} 5^{+}$and $\mathrm{CD} 34^{+}$cells are not detectable in skin postnatally until the first telogen phase, when and how HFSCs are established during embryonic hair development (reviewed in refs. 39, 40) were uncertain until 2008, when more recently identified stem cell markers and label-retaining studies were used to locate putative HFSCs during hair morphogenesis (18).

In these experiments, presumptive HFSCs were first detected in the suprabasal layer overlying the basal layer of hair germs. As in the adult, LRCs were shown to localize to the upper ORS during development and express several HFSC markers, including Sox9 $(18,20)$ and Tcf3 (ref. 41 and Figure 3). Additionally, loss of embryonic Sox9 expression in the epidermis resulted in the eventual loss of putative stem cell markers, as well as progressive loss of matrix cells during follicular development $(18,20)$. Although this study (18) suggests that Sox9 $9^{+}$putative stem cells are generated early during development and give rise to the bulge population (LRCs) postnatally, the lack of formal lineage tracing experiments precludes a definitive conclusion. Put another way, it remains to be determined whether Sox9-expressing cells in embryonic skin give rise to the Sox9 $9^{+}$cells in the bulge and/or to other stem cells that contribute to the sebaceous gland, infundibulum, and epidermis.

During development, melanoblasts migrate from the neural crest to colonize the forming hair follicle. Similar to HFSCs, follicular McSCs are established during hair follicle development and adopt a quiescent undifferentiated state in the presumptive bulge (42). TGF- $\beta$ receptor signaling in both embryonic and postnatal bulge McSCs is mediated by TGF- $\beta$ secreted within the bulge and is important for maintaining undifferentiated McSCs during the adult hair cycle (43). This study (43) brings a new perspective to our understanding of how cellular interactions within the niche can promote the establishment of McSCs during hair development and growth $(43,44)$.

\section{Neogenesis of the bulge in the adult}

In general, hair follicles develop de novo only during embryonic development, and new hair follicles do not form in postnatal skin. Therefore, skin diseases that result in loss of the HFSC compartment or of the entire follicle lead to permanent hair loss. Despite largely anecdotal data, the existence of de novo hair follicle regeneration in adult mammals was debated and essentially discounted (45-48). However, recent evidence has illuminated the potential for adult skin to regenerate entire skin appendages in 


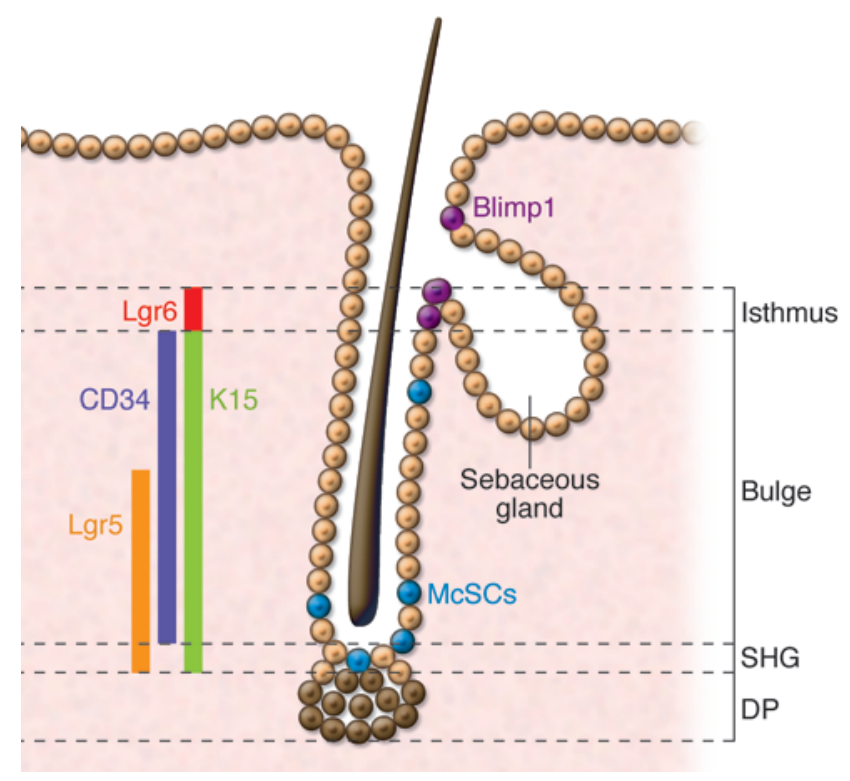

Figure 4

The geography of stem cells within the hair follicle. Several distinct and overlapping populations of cells have been identified within the telogen hair follicle. The cells that serve as a reservoir for the anagen hair follicle, including McSCs, reside in the sHG and bulge. The sHG sits above the DP during telogen and expresses specific markers (e.g., $\mathrm{P}$-cadherin), while the cells of the bulge upregulate bulge-specific markers (e.g., CD34, Nfatc1, S100A4). Some markers are expressed in both the sHG and bulge in an overlapping manner (e.g., K15, Lgr5). In the isthmus region (between the bulge and opening of the sebaceous gland into the pilary canal), there are other stem cell populations that maintain the sebaceous gland and/or epidermis. Lgr6 and Lrig1 are expressed in isthmus cells and give rise to the sebaceous gland and epidermis. In adult mouse hair follicles, Blimp1 cells are located near or at the opening of the sebaceous gland to the hair canal and appear to act as unipotent sebaceous gland progenitors.

vivo. Hair follicle regeneration following repair of large full-thickness wounds was definitively demonstrated in adult mice (49). In that study, nascent hair follicles displayed similar morphological and biochemical changes as those observed during embryonic hair development (49). In essence, hair follicle neogenesis represents a rare example of amphibian-like organ regeneration in mammals and demonstrates that embryonic developmental programs may be recapitulated in adult skin during wound healing.

In the study by Ito et al. (49), $\mathrm{K}^{+} 5^{+}$bulge-derived follicular cells did not significantly contribute to nascent hair follicles, although neogenic hair follicles generated new HFSCs that allowed them to cycle independently of surrounding follicles in intact skin. This suggests that neogenic follicles may be derived from either normally unipotent epidermal basal cells and/or another hair follicle population outside of the bulge/sHG. Lgr6 $^{+}$ cells represent a specific population of cells that reside within the isthmus above the bulge and contribute to the sebaceous gland and epidermis during homeostasis (24). Similar to K15-derived bulge cells, Lgr6-labeled cells mobilize to the epidermis following wounding. These Lgr6-derived cells were shown to durably contribute to wound-induced neogenic hair follicles, suggesting that some hair follicle cells can serve as precursors for neogenic hair follicles and neogenic HFSCs.

\section{Life outside the bulge}

Bulge and sHG cells of the telogen follicle typically give rise to the lower part of the hair follicle during homeostasis, suggesting that other stem cell populations in the hair follicle maintain the sebaceous gland, infundibulum, and epidermis in vivo. Several groups have defined other populations in the upper hair follicle that carry unique functions, underlining the heterogeneity of the HFSC niche.

The isthmus of the hair follicle extends between the telogen bulge region, typically defined by CD34/K15 expression, and the point where the sebaceous gland opens into the hair canal $(13,14)$. Studies have demonstrated that the isthmus of the hair follicle houses stem cells that contribute to other epithelial lineages in vivo $(21,23,24,50)$. Cells in this area express certain markers, including Lrig1, MTS24, Blimp-1, and Lgr6, that are not expressed in the bulge or sHG (Figure 4).

In the adult, $\operatorname{Lgr} 6^{+}$cells are confined to the central isthmus. Genetic lineage analysis showed that Lgr6-derived cells contribute to both the epidermis and sebaceous glands during homeostasis (24). However, the apparent dilution of label within the isthmus suggests that these cells may self-renew after migrating from their original residence. $\mathrm{Lgr6}^{+}$cells migrate out of the hair follicle to populate the epidermis soon after wounding. In contrast to K15derived cells that only transiently remain in the epidermis, Lgr6 lineage-labeled cells are maintained in the epidermis $(8,24)$. The intrinsic mechanisms that allow grr $^{+}$cells to provide self-renewing cells to the epidermis have yet to be examined. Similar to $\operatorname{Lgr}^{+}$ cells, Lrig1-expressing cells also localize to the isthmus and appear to contribute to the sebaceous gland and epidermis in vivo (23). These two isthmus populations may overlap or represent hierarchically related but distinct progenitors.

Recently, another subpopulation of cells was identified that is uniquely regulated by cutaneous sensory neurons (51). In the telogen hair follicle, the Hedgehog $(\mathrm{Hh})$ pathway transcriptional activator, Gli1, is expressed by cells that reside in the DP and sHG/lower bulge, as well as in a region that lies between the $\mathrm{CD} 34 / \mathrm{K} 5^{+}$bulge area and the Lrig1/MTS24 ${ }^{+}$isthmus (Figure 4 and ref. 51). Characterization of these cells showed that the uppermost population contributes to the anagen hair follicle and is self-renewing. Although coincident lineage labeling makes the precise long-term contribution of the uppermost $\mathrm{Gli1}^{+}$population to the hair follicle difficult to determine, this finding generates interesting questions regarding the requirement and function of this and other subpopulations in hair follicle regeneration. Furthermore, the study by Brownell et al. (51) showed that $\mathrm{Gli1} 1^{+}$upper bulge cells also contribute durably to the regenerated epidermis following wounding in a manner dependent upon nerve-derived extrinsic signals. It is unclear whether $\operatorname{Lgr}^{+}$ cells are similarly regulated by these signals and/or whether they overlap with Gli1 ${ }^{+}$cells.

In adult mouse skin, Blimp-1 is expressed in cells near the sebaceous gland opening and in differentiated cells of the hair and epidermis (21). Based on its expression pattern and the finding that embryonic Blimp-1 deletion in keratinocytes results in sebaceous gland hyperplasia, Blimp-1 appears to regulate unipotent progenitors of the sebaceous gland (21). In contrast, human embryonic and adult skin show Blimp-1 expression confined to differentiated cells of the hair follicle, sebaceous gland, and epidermis, suggesting a role for Blimp-1 in differentiated sebocytes rather than sebocyte progenitors in humans (52). 


\section{Stem cell interactions within the niche - another player}

Although most studies have focused on the regulation of follicular epithelial stem cells in hair regeneration, the bulge/sHG niche also maintains McSCs that are responsible for producing mature melanocytes during anagen (37). McSCs are coordinately activated together with HFSCs during anagen and populate the hair bulb with mature melanocytes that produce and transfer pigment to differentiating hair matrix cells, resulting in formation of a pigmented hair fiber. Studies demonstrated that loss of McSCs leads to hair graying (42). Also, bulge McSCs are implicated in repopulating epidermal melanocytes during the recovery process of vitiligo, a disease characterized by skin depigmentation due to a loss of mature skin melanocytes (53). How McSC behavior is coordinately regulated with HFSCs throughout the hair cycle has recently been examined. One study indicated that the maintenance and survival of McSCs in the hair follicle are not only regulated by intrinsic mechanisms, but also depend on the presence of HFSCs (43). Growing evidence shows that HFSCs secrete paracrine factors that activate signaling pathways in McSCs important for their growth and maintenance within this shared niche. TGF- $\beta$, Wnts, and endothelins that are secreted by HFSCs within the bulge/sHG have been shown to govern the behavior of McSCs during the hair cycle $(43,44)$.

These findings have uncovered new potential clinical targets to modulate McSC behavior for the treatment of pigmentation disorders. Most studies had focused on the reciprocal interactions between stem cells and inductive dermal cells (e.g., the DP) during hair follicle morphogenesis and regeneration. Further investigation of the heterotypic interactions between different stem cells may provide new insight into how distinct lineages coordinate their behavior during formation of pigmented hair. More generally, these studies address a fundamental question in developmental biology and regenerative medicine: how complex organs with multiple cell types can be regenerated in adult mammals (44).

\section{Clinical implications of HFSC biology}

HFSCs hold tremendous potential for the treatment of skin diseases. The realization that the HFSC niche is biochemically and functionally heterogeneous makes characterization of these distinct populations even more essential for our understanding of diverse skin diseases. For instance, certain forms of scarring alopecias (e.g., discoid lupus erythematosus, lichen planopilaris) are associated with inflammation of the follicular bulge region (54), resulting in permanent hair loss. In androgenetic alopecia (AGA), a non-scarring alopecia, hair follicles become miniaturized, and the number of follicular cells expressing high levels of K15 are unchanged compared to haired scalp. A specific progenitor subpopulation $\left(\mathrm{CD} 200^{+}\right.$, integrin $\left.\alpha 6^{+}\right)$in human hair follicles was recently identified and found to be diminished in hair follicles from balding scalp (55). This population lies within the sHG and bulge and is proliferatively more active than other $\mathrm{K}^{+} 5^{+}$HFSCs. An analogous population in mouse hair follicles also localized to the bulge and sHG and was multipotent in a reconstitution assay, underscoring the heterogeneity of the HFSC niche in both mice and humans (55). Due to a lack of available functional assays utilizing human cells, it is difficult to definitively determine whether loss or dysfunction of a specific cell population in humans is directly involved in pathogenesis. The development of assays to study the function of human hair stem cells ex vivo or in vitro is sorely needed.
As long-lived residents of the skin, skin stem cells sustain multiple genetic hits over the lifetime of the organism, and many skin tumors are thought to originate from epidermal stem cells. Basal cell carcinomas (BCCs) are the most common skin cancer in humans (56-58). Several studies have shown that BCCs are due to mutations (e.g., induced by UV irradiation or inherited) in genes involved in activation of the Hh pathway (e.g., Ptch1), and certain types of BCCs may arise from the hair follicle (reviewed in refs. 59,60). This conclusion is partly based upon their expression of hair follicle markers (e.g., K15) and the fact that Hh signaling is primarily active in hair follicles. However, in mice that express activating mutations in the Hh pathway within specific epithelial compartments, one study showed that BCC-like tumors were more frequently induced in the interfollicular epidermis rather than the hair follicle, including the bulge (61). This study proposed that BCCs arise within the epidermis and subsequently upregulate hair follicle markers such as K15 (61). However, one limitation to this study was the lack of histological description of the proliferative lesions observed. This is important, as several basaloid proliferations in humans are benign but share histological characteristics with BCCs. In contrast, Wang et al. used formal lineage tracing analysis in a BCC mouse model that activated Hh signaling following radiation exposure (62). This study showed that a large majority of induced BCC tumors arise from K15-derived bulge cells. Recently, further dissection of the role of Hh signaling in the pathogenesis of BCCs showed that constitutive Hh activation specifically in $\mathrm{K} 15^{+}$-derived follicular cells results in the generation of nodular subtypes of BCCs, whereas those arising from the epidermis preferentially result in the superficial subtype (63). This study also showed that the formation of follicular-derived BCCs is hair cycle dependent, suggesting that other signaling pathways may influence the growth of BCCs (63). Small-molecule inhibitors of the Hh pathway are currently being tested in patients with Ptch1 mutations (basal cell nevus syndrome) and hold promise for a novel treatment for this common cancer $(64,65)$.

In essence, the skin's integrity relies upon the preservation and activation of epidermal stem cells during homeostasis and following injury. Understanding the molecular signals that govern them will promote our understanding and treatment of skin diseases that result from their loss or dysfunction. The recent growth of HFSC studies in mice has set the direction and pace for future human studies and clinical research.

\section{Acknowledgments}

We are grateful to E. Hamburg, P. Rabbani, M. Takeo, and T. Andl for their discussion and review of this manuscript. We especially thank T. Andl for German translation of publications by P. Unna and P. Stöhr. P. Myung is supported by the Dermatology Foundation and NIH T32 training grant 5T32AR007569. M. Ito is supported by the Ellison Medical Foundation and NIH National Institute of Arthritis and Musculoskeletal and Skin Diseases grant 1R01AR059768-01A1.

Address correspondence to: Mayumi Ito, Departments of Dermatology and Cell Biology, New York University School of Medicine, 550 First Ave., Smilow Bldg, Rm 410, New York, New York 10016, USA. Phone: 212.263.9254; Fax: 212.263.5819; E-mail: mayumi. ito@nyumc.org. 
1. Mackenzie I. Relationship between mitosis and the ordered structure of the stratum corneum in mouse epidermis. Nature. 1970;226(5246):653-655.

2. Potten C. The epidermal proliferative unit: the possible role of the central basal cell. Cell Tissue Kinet. 1974;7(1):77-88.

3. Cotsarelis G. Epithelial stem cells: A folliculocen tric view. Jinvest Dermatol. 2006;126(7):1459-1468.

4. Paus R, Cotsarelis G. The biology of hair follicles. NEngl J Med. 1999;341(7):491-497.

5. Blanpain C, Lowry WE, Geoghegan A, Polak L, Fuchs E. Self-renewal, multipotency, and the existence of two cell populations within an epithelial stem cell niche. Cell. 2004;118(5):635-648.

6. Morris RJ, et al. Capturing and profiling adult hair follicle stem cells. Nat Biotechnol. 2004 22(4):411-417.

7. Oshima H, Rochat A, Kedzia C, Kobayashi K, Barrandon Y. Morphogenesis and renewal of hair follicles from adult multipotent stem cells. Cell. 2001; 104(2):233-245

8. Ito $\mathrm{M}$, et al. Stem cells in the hair follicle bulge contribute to wound repair but not to homeostasis of the epidermis. Nat Med. 2005;11(12):1351-1354.

9. Levy V, Lindon C, Harfe BD, Morgan BA. Distinct stem cell populations regenerate the follicle and interfollicular epidermis. Dev Cell. 2005;9(6):855-861.

10. Taylor G, Lehrer MS, Jensen PJ, Sun TT, Lavker RM. Involvement of follicular stem cells in forming not only the follicle but also the epidermis. Cell. 2000 102(4):451-461.

11. Tumbar T, et al. Defining the epithelial stem cell niche in skin. Science. 2004;303(5656):359-363

12. Cotsarelis G, Sun TT, Lavker RM. Label-retaining cells reside in the bulge area of pilosebaceous unit: implications for follicular stem cells, hair cycle, and skin carcinogenesis. Cell. 1990;61(7):1329-1337.

13. Lyle S, Christofidou-Solomidou M, Liu Y, Elder DE, Albelda S, Cotsarelis G. The C8/144B monoclona antibody recognizes cytokeratin 15 and defines the location of human hair follicle stem cells J Cell Sci. 1998;111(pt 21):3179-3188.

14. Trempus CS, et al. Enrichment for living murine keratinocytes from the hair follicle bulge with the cell surface marker CD34. J Invest Dermatol. 2003; 120(4):501-511.

15. Horsley V, Aliprantis AO, Polak L, Glimcher LH, Fuchs E. NFATc1 balances quiescence and proliferation of skin stem cells. Cell. 2008;132(2):299-310.

16. Jaks $\mathrm{V}$, et al. Lgr5 marks cycling, yet long-lived, hair follicle stem cells. Nat Genet. 2008;40(11):1291-1299.

17. Nguyen H, Rendl M, Fuchs E. Tcf3 governs stem cell features and represses cell fate determination in skin. Cell. 2006;127(1):171-183

18. Nowak JA, Polak L, Pasolli HA, Fuchs E. Hair follicle stem cells are specified and function in early skin morphogenesis. Cell Stem Cell. 2008;3(1):33-43.

19. Rhee H, Polak L, Fuchs E. Lhx2 maintains stem cell character in hair follicles. Science. 2006 312(5782):1946-1949.

20. Vidal VP, et al. Sox9 is essential for outer root sheath differentiation and the formation of the hair stem cell compartment. Curr Biol. 2005;15(15):1340-1351.

21 . Horsley V, et al. Blimp1 defines a progenitor population that governs cellular input to the sebaceous gland. Cell. 2006;126(3):597-609.

22. Ito M, Kizawa K, Toyoda M, Morohashi M. Labelretaining cells in the bulge region are directed to cell death after plucking, followed by healing from the surviving hair germ. J Invest Dermatol. 2002; 119(6):1310-1316

23. Jensen KB, et al. Lrig1 expression defines a distinct multipotent stem cell population in mammalian epidermis. Cell Stem Cell. 2009;4(5):427-439.

24. Snippert HJ, et al. Lgr6 marks stem cells in the hair follicle that generate all cell lineages of the skin. Science. 2010;327(5971):1385-1389.

25. Stohr P. Entwicklungsgeschichte des menschlichen wollhaares. Anat Hefte Abt 1. 1904;23:1-66.

26. Unna P. Beitrage zur histologie und entwicklungsgeschichte der menschlichen oberhaut und hrer anhangsgebilde. Arch mikroskop Anat Entwicklungsmech. 1876;12:665-741.

27. Madsen A. Studies on the "bulge" (wulst) in superficial basal epitheliomas. Arch Dermatol. 1964; 89:698-708.

28. Pinkus H. Embryology Of Hair. New York, New York, USA: Academy Press; 1958.

29. Liu Y, Lyle S, Yang Z, Cotsarelis G. Keratin 15 promoter targets putative epithelial stem cells in the hair follicle bulge. J Invest Dermatol. 2003; 121(5):963-968.

30. Chase HB, Rauch R, Smith VW. Critical stages of hair development and pigmentation in the mouse. Physiol Zool. 1951;24(1):1-8.

31. Silver AF, Chase HB. DNA synthesis in the adult hair germ during dormancy (telogen) and activation (early anagen). Dev Biol. 1970;21(3):440-451.

32. Silver AF, Chase HB, Arsenault CT. Early anagen initiated by plucking compared with early spontaneous anagen. In: Montagna W, Kenshalo DR, eds. Advances in Biology of Skin. Vol. 9. London, United Kingdom: Pergamon Press; 1967:265-289.

33. Ito M, Kizawa K. Expression of calcium-binding S100 proteins A4 and A6 in regions of the epithelial sac associated with the onset of hair follicle regeneration. J Invest Dermatol. 2001;116(6):956-963.

34. Ito M, Kizawa K, Hamada K, Cotsarelis G. Hair follicle stem cells in the lower bulge form the secondary germ, a biochemically distinct but functionally equivalent progenitor cell population, at the termination of catagen. Differentiation. 2004; 72(9-10):548-557.

35. Greco V, et al. A two-step mechanism for stem cell activation during hair regeneration. Cell Stem Cell. 2009; 4(2):155-169.

36. Zhang YV, Cheong J, Ciapurin N, McDermitt DJ, Tumbar T. Distinct self-renewal and differentiation phases in the niche of infrequently dividing hair follicle stem cells. Cell Stem Cell. 2009;5(3):267-278

37. Nishimura EK, et al. Dominant role of the niche in melanocyte stem-cell fate determination. Nature. 2002;416(6883):854-860

38. Hsu YC, Pasolli HA, Fuchs E. Dynamics between stem cells, niche, and progeny in the hair follicle. Cell. 2011;144(1):92-105.

39. Millar S. Molecular mechanisms regulating hair follicle development. J Invest Dermatol. 2002; 118(2):216-225.

40. Schmidt-Ullrich R, Paus R. Molecular principles of hair follicle induction and morphogenesis. Bioessays. 2005;27(3):247-261.

41. Merrill BJ, Gat U, DasGupta R, Fuchs E. Tcf3 and Lef1 regulate lineage differentiation of multipotent stem cells in skin. Genes Dev. 2001; 15(13):1688-1705

42. Nishimura EK, Granter SR, Fisher DE. Mechanisms of hair graying: incomplete melanocyte stem cell maintenance in the niche. Science. 2005; 307(5710):720-724

43. Tanimura $S$, et al. Hair follicle stem cells provide a functional niche for melanocyte stem cells. Cell Stem Cell. 2011;8(2):177-187.

44. Rabbani $\mathrm{P}$, et al. Coordinated activation of Wnt in epi- thelial and melanocyte stem cells initiates pigmented hair regeneration. Cell. 2011;145(6):941-955.

45. Billingham RE, Russell PS. Incomplete wound contracture and the phenomenon of hair neogenesis in rabbits' skin. Nature. 1956;177(4513):791-792.

46. Breedis C. Regeneration of hair follicles and sebaceous glands from the epithelium of scars in the rabbit. Cancer Res. 1954;14(8):575-579.

47. Kligman AM, Strauss JS. The formation of vellus hair follicles from human adult epidermis. J Invest Dermatol. 1956;27(1):19-23

48. Lacassagne A, Latarjet R. Action of methylcholanthrene on certain scars of the skin in mice. Cancer Res. 1946;6:183-188.

49. Ito M, et al. Wnt-dependent de novo hair follicle regeneration in adult mouse skin after wounding. Nature. 2007;447(7142):316-320.

50. Nijhof JG, et al. The cell-surface marker MTS24 identifies a novel population of follicular keratinocytes with characteristics of progenitor cells. Development. 2006;133(15):3027-3037.

51. Brownell I, Guevara E, Bai CB, Loomis CA, Joyner AL. Nerve-derived sonic hedgehog defines a niche for hair follicle stem cells capable of becoming epidermal stem cells. Cell Stem Cell. 2011;8(5):552-565.

52. Sellheyer K, Krahl D. Blimp-1: a marker of terminal differentiation but not of sebocytic progenitor cells. J Cutan Pathol. 2010;37(3):362-370.

53. Ortonne JP, MacDonald DM, Micoud A, Thivolet J. PUVA-induced repigmentation of vitiligo: a histochemical (split-DOPA) and ultrastructural study. Br J Dermatol. 1979;101(1):1-12.

54. Mobini N, Tam S, Kamino H. Possible role of the bulge region in the pathogenesis of inflammatory scarring alopecia: lichen planopilaris as the prototype. J Cutan Pathol. 2005;32(10):675-679.

55. Garza LA, et al. Bald scalp in men with androgenetic alopecia retains hair follicle stem cells but lacks CD200-rich and CD34-positive hair follicle progenitor cells. J Clin Invest. 2011;121(2):613-622.

56. Miller DL, Weinstock MA. Nonmelanoma skin cancer in the United States: incidence. J Am Acad Dermatol. 1994;30(5 pt 1):774-778.

57. Leiter U, Garbe C. Epidemiology of melanoma and nonmelanoma skin cancer--the role of sunlight. Adv Exp Med Biol. 2008;624:89-103

58. Rubin AI, Chen EH, Ratner D. Basal-cell carcinoma. N Engl J Med. 2005;353(21):2262-2269.

59. Epstein EH. Basal cell carcinomas: attack of the hedgehog. Nat Rev Cancer. 2008;8(10):743-754

60. Teglund S, Toftgard R. Hedgehog beyond medulloblastoma and basal cell carcinoma. Biochim Biophys Acta. 2010;1805(2):181-208

61 . Youssef KK, et al. Identification of the cell lineage at the origin of basal cell carcinoma. Nat Cell Biol. 2010; 12(3):299-305

62. Wang GY, Wang J, Mancianti ML, Epstein EH Jr. Basal cell carcinomas arise from hair follicle stem cells in Ptch1(+/-) mice. Cancer Cell. 2011; 19(1):114-124

63. Grachtchouk M, et al. Basal cell carcinomas in mice arise from hair follicle stem cells and multiple epithelial progenitor populations. J Clin Invest. 2011; 121(5):1768-1781.

64. Gupta S, Takebe N, Lorusso P. Targeting the Hedgehog pathway in cancer. Ther Adv Med Oncol. 2010; 2(4):237-250.

65. Kiesslich T, et al. Current status of therapeutic targeting of developmental signalling pathways in oncology [published online ahead of print May 24, 2011]. Curr Pharm Biotechnol. doi:10.2174/1389211 216050742010 